\title{
Efeito de diferentes diluidores sobre a viabilidade espermática pós-descongelação de sêmen eqüino
}

\author{
[Effect of differents freezing extenders on post thaw equine spermatozoa viability] \\ P.P.N. Snoeck ${ }^{1}$, M. Henry ${ }^{2}$, M.I.V. Melo ${ }^{3}$ \\ ${ }^{1}$ Universidade Estadual de Santa Cruz \\ Rod. Ilhéus-Itabuna, km 16, \\ 45.650-000 - Ilhéus, BA \\ ${ }^{2}$ Escola de Veterinária - UFMG - Belo Horizonte, MG \\ ${ }^{3}$ Pontifícia Universidade Católica - Belo Horizonte, MG
}

\section{RESUMO}

Estudou-se o efeito de diferentes concentrações de gema de ovo, açúcares e tampões nos diluidores sobre a viabilidade espermática pós-descongelação de sêmen eqüino. Os diluidores foram: D1 $=10 \%$ de gema de ovo e acréscimo de $50 \%$ de lactose e glicose-EDTA em relação ao D5; D2=10\% de gema de ovo e redução de $50 \%$ de lactose e glicose-EDTA em relação ao D5; D3=20\% de gema de ovo e acréscimo de $50 \%$ de lactose e glicose-EDTA em relação ao D5; D4= 20\% de gema de ovo e redução de 50\% de lactose e glicose-EDTA em relação ao D5; D5= 20\% de gema de ovo e composição de lactose e glicose-EDTA segundo Martin et al. (1979). Cinco ejaculados de seis garanhões foram utilizados para testar os diluidores contendo diferentes agentes crioprotetores: $3,5 \%$ de etilenoglicol e 5\% de acetamida. Após diluição final nos diferentes diluidores, o sêmen foi submetido a diferentes protocolos de congelação: com e sem resfriamento prévio. Após descongelação em banho-maria a $75^{\circ} \mathrm{C}$, avaliaram-se a motilidade, o vigor, e a integridade estrutural e funcional das membranas espermáticas. A redução na concentração de gema de ovo de $20 \%$ para $10 \%$, nos diluidores contendo a mesma concentração de açúcares e tampões, não alterou a capacidade crioprotetora dos diluidores com etilenoglicol ou acetamida. $\mathrm{O}$ aumento na concentração de açúcares e tampões no diluidor lactose-EDTA-gema de ovo não acrescentou efeito crioprotetor ao etilenoglicol e acetamida. Diluidores com maior osmolaridade tenderam a preservar melhor o sêmen eqüino congelado com etilenoglicol ou acetamida. Os melhores resultados de viabilidade espermática, pós-descongelação, foram para o sêmen congelado nos diluidores D1, D3 e D5 $(\mathrm{P}<0,05)$.

Palavras-chave: eqüino, sêmen, criopreservação, diluidor

\begin{abstract}
The effect of different egg yolk, sugars and buffering systems concentrations in the freezability of differents equine semen extenders was studied. Extenders were: D1 $10 \%$ of egg yolk added of more $50 \%$ over the lactose and glicose-EDTA levels found in D5; D2 $=10 \%$ of egg yolk and reduction of 50\% over the lactose and glucose-EDTA levels in D5; D3=20\% of egg yolk added of more 50\% over the lactose and glucose-EDTA levels in D5; D4=20\% of egg yolk and reduction of 50\% over the lactose and glucose-EDTA levels in D5; D5 $=20 \%$ of egg yolk and lactose and glucose-EDTA according to Martin et al. (1979). Five ejaculates of six stallions were used to test extenders using alternatively two differents cryoprotectants agents: $3.5 \%$ ethylene glycol and 5\% acetamide. After the final dilution, the semen was submitted to differents freezing rates. Straws were thawed at $75^{\circ} \mathrm{C}$ during 7 seconds, followed by immersion at $37^{\circ} \mathrm{C}$ during 5 seconds. After thawing motility, vigour, structural and functional integrity of the membrane of the spermatozoa were evaluated. The reduction in the egg yolk concentration from $20 \%$ to $10 \%$, in extenders with the same concentration of sugars and buffering systems, did not modify the crioprotectant capacity of the extender with ethylene glycol or acetamide. The increase in the concentration of sugars and buffering systems in the lactose-EDTA-egg yolk extender did not add crioprotectant effect to ethylene glycol and acetamide. Extenders with high osmolarity
\end{abstract}

Recebido em 22 de julho de 2005

Aceito em 30 de novembro de 2006

E-mail: paolasnoeck@uesc.br 
had tended to better preserve the frozen equine semen with ethylene glycol or acetamide. Better results of spermatic viability, after thawing, were obtained when semen was diluted in D1, D3 and D5 extender $(P<0.05)$.

Keywords: equine, semen, cryopreservation, extender

\section{INTRODUÇÃO}

A composição dos diluidores de congelação de sêmen pode afetar os resultados do processo de criopreservação. A proteção das células durante a fase de desidratação que ocorre durante a congelação e a estabilização da bicamada lipídica, como o impedimento da fusão de partículas intramembranosas, depende das substâncias que compõem o diluidor (Crowe et al., 1987).

Alguns diluidores são mais utilizados para o processo de congelação de sêmen eqüino, dentre eles estão o diluidor Naumienkov e Romankova (Tischner, 1979); o INRA 82, com várias concentrações de gema de ovo e glicerol (Palmer, 1984); o diluidor proposto por Martin et al. (1979), formulado com lactose-EDTA-gema de ovo e o diluidor Kenney, acrescido de gema de ovo e glicerol (Burns, 1992; Burns e Reasner, 1995). A maioria dos diluidores possui em sua formulação açúcares, eletrólitos, gema de ovo; em concentração variável de 1,6 a $20 \%$, e glicerol, variando entre 2,5 e $5 \%$. Os diluidores como o INRA 82 e o Kenney, além dos componentes anteriormente citados, possuem leite na sua composição.

Outros meios de congelação incluem o meio Gent, à base de açúcares, leite, tampões, 5\% de gema de ovo e 5\% de glicerol; o meio MP-50, constituído de gema de ovo, leite desnatado, Dulbeccos Basal Medium Eagle (BME) e uma associação de glicerol e dimetilformamida (Gomes et al., 2002; Papa et al., 2002); o meio glicina gema de ovo (Papa et al., 1993); e o meio BME-adaptado, formulado a partir de uma associação de meios: Merk-gema (33\%), Kenney $(31,7 \%)$ e Dulbeccos Basal Medium Eagle $(31,7 \%)$ (Neves Neto et al., 1999). Considerando esses dados, fica evidente, na literatura consultada, que uma grande diversidade de diluidores de congelação tem sido testada para o sêmen eqüino. Essa variabilidade de composição é indicativa de que ainda não foi encontrado um diluidor que preserve a viabilidade espermática pós-descongelação para a maioria dos garanhões. Uma das causas que leva à existência dessa diversidade de meios diluidores deve estar ligada ao fato de existir uma variabilidade na sensibilidade espermática à congelação/ descongelação entre garanhões e/ou entre ejaculados de um mesmo garanhão (Samper et al., 1991).

Foi objetivo deste experimento testar a eficácia de crioprotetores intracelulares alternativos e o efeito da osmolaridade utilizando-se diferentes meios de congelação à base de lactose-EDTAgema de ovo, sobre a viabilidade espermática pós-descongelação de sêmen eqüino.

\section{MATERIAL E MÉTODOS}

Foram utilizados seis garanhões, sendo cinco da raça Mangalarga Marchador e um Bretão, com idades variando entre três e 15 anos. Previamente ao processamento do sêmen, os garanhões foram submetidos a uma colheita de sêmen (vagina artificial) diária, por sete dias consecutivos, para equilibrar as reservas extragonadais. Cinco ejaculados de cada garanhão foram submetidos à congelação, mantendo-se um intervalo entre colheitas, de três dias.

Imediatamente após a colheita, foram avaliados a motilidade total e progressiva e vigor espermáticos (Manual..., 1998) e realizado o teste hiposmótico (HO), para avaliar a integridade funcional da membrana plasmática (Melo e Henry, 1999). Após análise, somente ejaculados que apresentaram motilidade espermática progressiva mínima de $50 \%$ foram submetidos à congelação. Na seqüência, o sêmen foi diluído 1:2 no diluidor de centrifugação glicose-EDTA proposto por Martin et al. (1979), sendo centrifugado a $400 \mathrm{xg}$ por 10 minutos. Após a centrifugação, foi mantido de $10 \%$ a $20 \%$ de plasma seminal, e a ressuspensão dos espermatozóides nos meios de congelação foi ajustada de forma a obter 100 milhões de espermatozóides/ml. 
As amostras foram, então, submetidas a duas curvas de congelação. A primeira foi denominada de curva rápida com resfriamento prévio lento; para isso, as amostras foram resfriadas $\left(-0,13^{\circ} \mathrm{C} / \mathrm{min}\right) \mathrm{em}$ geladeira doméstica por duas horas e meia até a temperatura de $5^{\circ} \mathrm{C}$; depois, o sêmen foi envasado em palhetas de $0,5 \mathrm{ml}$ e submetido à congelação, em caixa de isopor, $3 \mathrm{~cm}$ acima da fase líquida do nitrogênio, durante 10 minutos; durante o envase, o sêmen permaneceu em sacos plásticos estéreis submersos em água a $5^{\circ} \mathrm{C}$, no intuito de evitar grandes variações de temperatura. A segunda curva de congelação foi denominada de curva rápida sem resfriamento; nesse caso, o sêmen foi envasado em palhetas de $0,5 \mathrm{ml}$, imediatamente após a diluição final, e congelado como descrito anteriormente. As amostras foram, por conseguinte, armazenadas em botijão até a descongelação e avaliação.

A descongelação das amostras foi realizada em banho-maria a $75^{\circ} \mathrm{C}$, por sete segundos, seguida de imersão em banho-maria a $37^{\circ} \mathrm{C}$, por cinco segundos. Após descongelação, avaliou-se a motilidade progressiva, o vigor, a morfologia espermática, a integridade estrutural das membranas plasmática e acrossomal por meio de sondas fluorescentes (Harrison e Vickers, 1990, modificado por Zúccari, 1998), e a integridade funcional da membrana plasmática dos espermatozóides por meio do teste HO (Melo e Henry, 1999).

Neste experimento, foram testados cinco diluidores à base de lactose-EDTA-gema de ovo com diferentes concentrações de crioprotetores intracelulares, gema de ovo, açúcares e tampões. Para tanto, considerou-se como controle o meio de congelação D5 constituído de lactose-EDTA-gema de ovo descrito por Martin et al. (1979). O D1 foi preparado com $10 \%$ de gema de ovo e acréscimo de $50 \%$ de lactose e glicose-EDTA em relação ao D5; o D2 apresentou $10 \%$ de gema de ovo e redução de $50 \%$ de lactose e glicose-EDTA em relação ao D5; o D3 foi formulado com $20 \%$ de gema de ovo e acréscimo de $50 \%$ de lactose e glicose-EDTA em relação ao D5; e o D4 com $20 \%$ de gema de ovo e redução de $50 \%$ de lactose e glicose-EDTA em relação ao D5. Em cada meio diluidor base, o glicerol foi substituído por 3,5\% de etilenoglicol ou $5 \%$ de acetamida sempre associada a $0,5 \%$ de metilcelulose e $0,165 \mathrm{~g} / 100 \mathrm{ml}$ de trealose (Tab.1).

Tabela 1. Composição dos cinco diluidores de congelação à base de lactose-EDTA-gema de ovo usados para sêmen eqüino

\begin{tabular}{|c|c|c|c|c|c|c|c|c|c|c|}
\hline & D1 & & $\overline{\mathrm{D} 2}$ & & D3 & & D4 & & $\overline{\mathrm{D} 5}$ & \\
\hline Crioprotetor/Reagente & $\mathrm{E} 3,5 \%$ & $\mathrm{~A} 5 \%$ & $\mathrm{E} 3,5 \%$ & $\mathrm{~A} \%$ & $\mathrm{E} 3,5 \%$ & $\mathrm{~A} 5 \%$ & E3,5\% & $\mathrm{A} 5 \%$ & $\mathrm{E} 3,5 \%$ & A5\% \\
\hline Lactose $50 \mathrm{ml}$ & $16,5 \%$ & $16,5 \%$ & $5,5 \%$ & $5,5 \%$ & $16,5 \%$ & $16,5 \%$ & $5,5 \%$ & $5,5 \%$ & $11 \%$ & $11 \%$ \\
\hline Glicose-EDTA & $-(25$ & $\mathrm{ml})$ : & & & & & & & & \\
\hline Glicose (g) & 9 & 9 & 3 & 3 & 9 & 9 & 3 & 3 & 6 & 6 \\
\hline EDTA $(\mathrm{g})$ & 0,555 & 0,555 & 0,185 & 0,185 & 0,555 & 0,555 & 0,185 & 0,185 & 0,370 & 0,370 \\
\hline Citrato de sódio (g) & 0,560 & 0,560 & 0,188 & 0,188 & 0,560 & 0,560 & 0,188 & 0,188 & 0,375 & 0,375 \\
\hline $\begin{array}{l}\text { Bicarbonato de sódio } \\
(\mathrm{g})\end{array}$ & 0,18 & 0,18 & 0,06 & 0,06 & 0,18 & 0,18 & 0,06 & 0,06 & 0,12 & 0,12 \\
\hline Estreptomicina (g) & 0,05 & 0,05 & 0,05 & 0,05 & 0,05 & 0,05 & 0,05 & 0,05 & 0,05 & 0,05 \\
\hline Penicilina (UI) & 50 & 50 & 50 & 50 & 50 & 50 & 50 & 50 & 50 & 50 \\
\hline Água (ml) & 100 & 100 & 100 & 100 & 100 & 100 & 100 & 100 & 100 & 100 \\
\hline Gema de ovo & $10 \mathrm{ml}$ & $10 \mathrm{ml}$ & $10 \mathrm{ml}$ & $10 \mathrm{ml}$ & $20 \mathrm{ml}$ & $20 \mathrm{ml}$ & $20 \mathrm{ml}$ & $20 \mathrm{ml}$ & $20 \mathrm{ml}$ & $20 \mathrm{ml}$ \\
\hline Equex STM & $0,5 \mathrm{ml}$ & $0,5 \mathrm{ml}$ & $0,5 \mathrm{ml}$ & $0,5 \mathrm{ml}$ & $0,5 \mathrm{ml}$ & $0,5 \mathrm{ml}$ & $0,5 \mathrm{ml}$ & $0,5 \mathrm{ml}$ & $0,5 \mathrm{ml}$ & $0,5 \mathrm{ml}$ \\
\hline Crioprotetor & $3,5 \mathrm{ml}$ & $5 \mathrm{~g}$ & $3,5 \mathrm{ml}$ & $5 \mathrm{~g}$ & $3,5 \mathrm{ml}$ & $5 g$ & $3,5 \mathrm{ml}$ & $5 \mathrm{~g}$ & $3,5 \mathrm{ml}$ & $5 \mathrm{~g}$ \\
\hline Metilcelulose(g) & - & 0,5 & - & 0,5 & - & 0,5 & - & 0,5 & - & 0,5 \\
\hline Trealose (g) & - & 0,165 & - & 0,165 & - & 0,165 & - & 0,165 & - & 0,165 \\
\hline
\end{tabular}

D1:10\% de gema de ovo e acréscimo de 50\% de lactose e glicose-EDTA em relação ao D5; D2:10\% de gema de ovo e redução de 50\% de lactose e glicose-EDTA em relação ao D5; D3:20\% de gema de ovo e acréscimo de 50\% de lactose e glicose-EDTA em relação ao D5; e D4:20\% de gema de ovo e redução de 50\% de lactose e glicose-EDTA em relação ao D5; D5:20\% de gema de ovo e composição de lactose e de glicose-EDTA, segundo Martin et al. (1979). Crioprotetores intracelulares: etilenoglicol (E 3,5\%) e acetamida (A 5\%). Equex STM - Nova Chemical Sales, INC. 
A osmolaridade ${ }^{1}$ e o $\mathrm{pH}^{2}$ dos meios de congelação estão na Tab 2.

O delineamento experimental foi de parcelas subdivididas. Os diluidores foram considerados as parcelas, e o fatorial (dois crioprotetores $\mathrm{x}$ duas curvas de congelação) foi considerado a subparcela. $\mathrm{O}$ garanhão foi considerado como bloco, e os diferentes ejaculados de um mesmo garanhão foram selecionados ao acaso para serem congelados em diluidores distintos, para que todos os tratamentos (diluidor $\mathrm{x}$ crioprotetor intracelular $\mathrm{x}$ curva de congelação) fossem testados. O efeito dos fatores composição do meio diluidor, crioprotetor e curva de congelação foi avaliado via análise de variância. As médias obtidas para os diferentes parâmetros (motilidade, integridade estrutural das membranas plasmática e acrossomal e integridade funcional da membrana plasmática dos espermatozóides) foram comparadas pelo teste Duncan. As variáveis que apresentaram valores percentuais com grande amplitude de variação foram transformadas para ângulo, correspondendo a arco-seno $\sqrt{ } \mathrm{X} / 100$. A variável vigor, que não apresentou distribuição normal foi comparada pelo teste de Kruskal - Wallis (Sampaio, 1998). Na análise, foi utilizado o programa estatístico SAEG (Sistema..., 1997).

Tabela 2. Meios diluidores de congelação de sêmen eqüino: osmolaridade e pH

\begin{tabular}{lllll}
\hline Diluidor & \multicolumn{1}{c}{ Crioprotetor } & $\begin{array}{c}\text { Osmolaridade com } \\
\text { crioprotetor }\end{array}$ & $\begin{array}{c}\text { Osmolaridade sem } \\
\text { crioprotetor }\end{array}$ & $\mathrm{pH}$ \\
\hline D1 & Etilenoglicol 3,5\% & $810 \mathrm{mOsmol} / 1$ & $580 \mathrm{mOsmol} / 1$ & 6,50 \\
& Acetamida 5\% & $925 \mathrm{mOsmol} / 1$ & $606 \mathrm{mOsmol} / 1$ & 6,86 \\
\hline D2 & Etilenoglicol 3,5\% & $685 \mathrm{mOsmol} / 1$ & $195 \mathrm{mOsmol} / 1$ & 6,57 \\
& Acetamida 5\% & $880 \mathrm{mOsmol} / 1$ & $220 \mathrm{mOsmol} / 1$ & 6,27 \\
\hline D3 & Etilenoglicol 3,5\% & $780 \mathrm{mOsmol} / 1$ & $575 \mathrm{mOsmol} / 1$ & 6,40 \\
& Acetamida 5\% & $950 \mathrm{mOsmol} / 1$ & $692 \mathrm{mOsmol} / 1$ & 6,40 \\
\hline D4 & Etilenoglicol 3,5\% & $555 \mathrm{mOsmol} / 1$ & $207 \mathrm{mOsmol} / 1$ & 6,49 \\
& Acetamida 5\% & $950 \mathrm{mOsmol} / 1$ & $257 \mathrm{mOsmol} / 1$ & 6,56 \\
\hline D5 & Etilenoglicol 3,5\% & $1130 \mathrm{mOsmol} / 1$ & $400 \mathrm{mOsmol} / 1$ & 6,48 \\
& Acetamida 5\% & $1480 \mathrm{mOsmol} / 1$ & $445 \mathrm{mOsmol} / 1$ & 6,38 \\
\hline
\end{tabular}

D1:10\% de gema de ovo e acréscimo de 50\% de lactose e glicose-EDTA; D2:10\% de gema de ovo e redução de 50\% de lactose e glicose-EDTA; D3:20\% de gema de ovo e acréscimo de 50\% de lactose e glicose-EDTA; e D4:20\% de gema de ovo e redução de 50\% de lactose e glicose-EDTA do que o D5; D5:20\% de gema de ovo e composição de lactose e de glicose-EDTA, segundo Martin et al. (1979).

\section{RESULTADOS}

Os resultados de motilidade espermática progressiva e vigor para cada tratamento estão nas Tab. 3 e 4, respectivamente. Em comparações feitas entre crioprotetores em cada diluidor (D1-D5), observou-se que o crioprotetor acetamida preservou melhor a motilidade do que o etilenoglicol nos diluidores D1 e D3, independente da curva de congelação utilizada. Esse crioprotetor também preservou melhor o vigor, quando comparado à amostra congelada com resfriamento prévio em geladeira no diluidor D3, contendo etilenoglicol
$(\mathrm{P}<0,05)$. Nos diluidores $\mathrm{D} 2$ e $\mathrm{D} 4$, não foi observada qualquer diferença entre os crioprotetores e as curvas utilizadas $(\mathrm{P}>0,05)$. No D5, a motilidade pós-descongelação superior foi no diluidor com o crioprotetor acetamida, quando utilizado o resfriamento lento antes da congelação $(\mathrm{P}<0,05)$. $\mathrm{O}$ vigor espermático pós-descongelação foi a única característica que apresentou diferença entre os diluidores (D1-D5) em comparação feita independente do crioprotetor intracelular e da curva de congelação. O mesmo foi menor nas amostras submetidas à congelação em meio diluidor D2 comparado aos meios D1, D3 e D5 $(\mathrm{P}<0,05)$.

\footnotetext{
${ }^{1} \mu$ OSMETTE TM, Model 5004 - Automatic Osmometer-

Natick MA - USA

${ }^{2}$ pHmetro DM 20 - Digimed
} 
Tabela 3. Motilidade espermática (\%) do sêmen de garanhões após a descongelação utilizando diferentes protocolos de congelação (média de seis garanhões)

\begin{tabular}{ccccccc}
\multirow{2}{*}{ Crioprotetor } & Curva de & \multicolumn{5}{c}{ Diluidor } \\
\cline { 3 - 7 } & congelação & D1 & D2 & D3 & D4 & D5 \\
\hline Etilenoglicol 3,5\% & CR & $21,3 \pm 7,1 \mathrm{aB}$ & $13,3 \pm 7,0 \mathrm{aA}$ & $19,0 \pm 7,9 \mathrm{aB}$ & $16,6 \pm 5,9 \mathrm{aA}$ & $37,9 \pm 15,5 \mathrm{aB}$ \\
& $\mathrm{SR}$ & $23,8 \pm 10,0 \mathrm{aB}$ & $13,3 \pm 8,8 \mathrm{aA}$ & $24,8 \pm 13,3 \mathrm{aB}$ & $17,1 \pm 8,0 \mathrm{aA}$ & $45,8 \pm 18,3 \mathrm{aAB}$ \\
Acetamida 5\% & $\mathrm{CR}$ & $30,3 \pm 15,3 \mathrm{aA}$ & $16,3 \pm 8,0 \mathrm{aA}$ & $40,4 \pm 14,4 \mathrm{aA}$ & $15,4 \pm 8,1 \mathrm{aA}$ & $49,6 \pm 8,6 \mathrm{aA}$ \\
& $\mathrm{SR}$ & $34,0 \pm 15,1 \mathrm{aA}$ & $17,1 \pm 12,0 \mathrm{aA}$ & $38,3 \pm 13,5 \mathrm{aA}$ & $18,8 \pm 7,5 \mathrm{aA}$ & $42,5 \pm 15,5 \mathrm{aAB}$ \\
\hline
\end{tabular}

Médias seguidas por letras minúsculas distintas na linha diferem entre si $(\mathrm{P}<0,05)$.

Médias seguidas por letras maiúsculas distintas na coluna diferem entre si $(\mathrm{P}<0,05)$.

CR:congelação rápida com resfriamento lento; SR:congelação rápida sem resfriamento lento. D1:10\% de gema de ovo e acréscimo de $50 \%$ de açúcares e tampões em relação ao D5; D2:10\% de gema de ovo e redução de $50 \%$ de açúcares e tampões em relação ao D5; D3:20\% de gema de ovo e acréscimo de 50\% de açúcares e tampões em relação ao D5; D4:20\% de gema de ovo e redução de $50 \%$ de açúcares e tampões em relação ao D5; D5:meio diluidor semelhante ao proposto por Martin et al. (1979), com 20\% de gema de ovo, lactose-glicose-EDTA.

Tabela 4. Vigor do movimento espermático (escala de 0 a 5) do sêmen de garanhões após a descongelação utilizando diferentes protocolos de congelação (média de seis garanhões)

\begin{tabular}{ccccccc}
\hline \multirow{2}{*}{ Crioprotetor } & Curva de & \multicolumn{5}{c}{ Diluidor } \\
\cline { 3 - 7 } & congelação & D1 & D2 & D3 & D4 & D5 \\
\hline Etilenoglicol 3,5\% & $\mathrm{CR}$ & $2,2 \pm 0,4 \mathrm{aA}$ & $1,4 \pm 0,5 \mathrm{bA}$ & $1,8 \pm 0,5 \mathrm{aB}$ & $1,8 \pm 0,8 \mathrm{abA}$ & $2,3 \pm 0,4 \mathrm{aA}$ \\
& $\mathrm{SR}$ & $2,3 \pm 0,6 \mathrm{aA}$ & $1,3 \pm 0,5 \mathrm{bA}$ & $2,6 \pm 0,7 \mathrm{aA}$ & $1,8 \pm 0,8 \mathrm{abA}$ & $2,4 \pm 0,7 \mathrm{aA}$ \\
Acetamida $5 \%$ & $\mathrm{CR}$ & $2,3 \pm 0,6 \mathrm{aA}$ & $1,6 \pm 0,5 \mathrm{bA}$ & $2,6 \pm 0,6 \mathrm{aA}$ & $1,7 \pm 0,4 \mathrm{abA}$ & $2,5 \pm 0,4 \mathrm{aA}$ \\
& $\mathrm{SR}$ & $2,5 \pm 0,5 \mathrm{aA}$ & $1,3 \pm 0,5 \mathrm{bA}$ & $2,7 \pm 0,5 \mathrm{aA}$ & $2,3 \pm 0,4 \mathrm{abA}$ & $2,6 \pm 0,5 \mathrm{aA}$ \\
\hline
\end{tabular}

Médias seguidas por letras minúsculas distintas na linha diferem entre si $(\mathrm{P}<0,05)$.

Médias seguidas por letras maiúsculas distintas na coluna diferem entre si $(\mathrm{P}<0,05)$.

CR:congelação rápida com resfriamento lento; SR:congelação rápida sem resfriamento lento. D1:10\% de gema de ovo e acréscimo de $50 \%$ de açúcares e tampões em relação ao D5; D2:0\% de gema de ovo e redução de 50\% de açúcares e tampões em relação ao D5; D3:20\% de gema de ovo e acréscimo de 50\% de açúcares e tampões em relação ao D5; D4:20\% de gema de ovo e redução de $50 \%$ de açúcares e tampões em relação ao D5; D5:meio diluidor semelhante ao proposto por Martin et al. (1979), com 20\% de gema de ovo, lactose-glicose-EDTA.

$\mathrm{Na}$ Tab. 5, apresenta-se a porcentagem de espermatozóides com membranas plasmática e acrossomal estruturalmente íntegras, avaliadas por meio das sondas fluorescentes após a descongelação. Os meios diluidores D3 e D5, com utilização de curva de congelação com resfriamento prévio, independente do uso do etilenoglicol ou acetamida como crioprotetores, preservaram melhor a integridade das membranas plasmática e acrossomal dos espermatozóides que os diluidores D2 e D4 $(\mathrm{P}<0,05)$. Os diluidores D1, D3 e D5, contendo o crioprotetor etilenoglicol a $3,5 \%$ e submetidos a congelação rápida sem resfriamento prévio, preservaram melhor a integridade estrutural das membranas espermáticas que os diluidores D2 e D4 $(\mathrm{P}<0,05)$. O diluidor D3 contendo o crioprotetor acetamida 5\% preservou melhor a integridade estrutural das membranas dos espermatozóides durante a criopreservação que os diluidores D2 e D4 $(\mathrm{P}<0,05)$, após o sêmen ser congelado sem resfriamento prévio. Em cada diluidor (D1-D5), não houve efeito do crioprotetor intracelular e curva de congelação sobre a integridade das membranas plasmática e acrossomal pós-descongelação $(\mathrm{P}>0,05)$.

Os resultados do teste hiposmótico são apresentados na Tab. 6. A única diferença observada foi entre os diluidores D2 e D5 contendo $3,5 \%$ de etilenoglicol quando congelados sem resfriamento prévio. A porcentagem de espermatozóides reativos ao teste hiposmótico pós-descongelação foi superior no D5 do que no D2 $(\mathrm{P}<0,05)$. Em cada diluidor, separadamente, não houve diferença entre os crioprotetores e as curvas de congelação na porcentagem de espermatozóides com integridade funcional da membrana plasmática $(\mathrm{P}>0,05)$. 
Tabela 5. Porcentagem de espermatozóides eqüinos submetidos a diferentes protocolos de criopreservação com membranas plasmática e acrossomal íntegras, avaliadas por meio das sondas fluorescentes, após a descongelação

\begin{tabular}{ccccccc}
\hline \multirow{2}{*}{ Crioprotetor } & Curva de & \multicolumn{5}{c}{ Diluidor } \\
\cline { 3 - 7 } & congelação & D1 & D2 & D3 & D4 \\
\hline \multirow{2}{*}{ Etilenoglicol 3,5\% } & CR & $31,0 \pm 11,9 \mathrm{abA}$ & $10,3 \pm 5,9 \mathrm{cA}$ & $46,8 \pm 21,9 \mathrm{aA}$ & $20,8 \pm 9,1 \mathrm{bA}$ & $43,3 \pm 14,2 \mathrm{aA}$ \\
& $\mathrm{SR}$ & $39,3 \pm 16,3 \mathrm{aA}$ & $14,5 \pm 12,3 \mathrm{bA}$ & $48,2 \pm 16,1 \mathrm{aA}$ & $23,0 \pm 10,4 \mathrm{bA}$ & $38,7 \pm 13,2 \mathrm{aA}$ \\
& $\mathrm{CR}$ & $33,7 \pm 12,8 \mathrm{abA}$ & $19,3 \pm 15,8 \mathrm{cA}$ & $41,7 \pm 11,5 \mathrm{aA}$ & $25,7 \pm 13,3 \mathrm{bcA}$ & $45,0 \pm 13,8 \mathrm{aA}$ \\
Acetamida 5\% & SR & $35,3 \pm 20,3 \mathrm{abA}$ & $22,3 \pm 14,4 \mathrm{bA}$ & $48,0 \pm 19,8 \mathrm{aA}$ & $23,3 \pm 10,3 \mathrm{bA}$ & $35,3 \pm 14,7 \mathrm{abA}$ \\
\hline
\end{tabular}

Médias seguidas por letras minúsculas distintas na linha diferem entre si $(\mathrm{P}<0,05)$.

Médias seguidas por letras maiúsculas distintas na coluna diferem entre si $(\mathrm{P}<0,05)$.

CR:congelação rápida com resfriamento lento; SR:congelação rápida sem resfriamento lento. D1:10\% de gema de ovo e acréscimo de $50 \%$ de açúcares e tampões em relação ao D5; D2:10\% de gema de ovo e redução de 50\% de açúcares e tampões em relação ao D5; D3:20\% de gema de ovo e acréscimo de 50\% de açúcares e tampões em relação ao D5; D4:20\% de gema de ovo e redução de 50\% de açúcares e tampões em relação ao D5; D5:meio diluidor semelhante ao proposto por Martin et al. (1979), com 20\% de gema de ovo, lactose-glicose-EDTA.

Tabela 6. Porcentagem de espermatozóides eqüinos reativos ao teste hiposmótico após a descongelação utilizando-se diferentes protocolos de criopreservação

\begin{tabular}{lcccccc}
\hline \multirow{2}{*}{ Crioprotetor } & Curva de & \multicolumn{5}{c}{ Diluidor } \\
\cline { 3 - 7 } & congelação & D1 & D2 & D3 & D4 & D5 \\
\hline \multirow{2}{*}{ Etilenoglicol 3,5\% } & CR & $18,8 \pm 10,1 \mathrm{aA}$ & $2,8 \pm 3,8 \mathrm{aA}$ & $20,2 \pm 19,5 \mathrm{aA}$ & $3,0 \pm 4,7 \mathrm{aA}$ & $34,3 \pm 20,2 \mathrm{aA}$ \\
& $\mathrm{SR}$ & $17,3 \pm 15,0 \mathrm{abA}$ & $0,0 \pm 0,0 \mathrm{bA}$ & $19,0 \pm 17,4 \mathrm{abA}$ & $3,0 \pm 3,7 \mathrm{abA}$ & $30,0 \pm 14,4 \mathrm{aA}$ \\
\multirow{2}{*}{ Acetamida 5\% } & $\mathrm{CR}$ & $17,3 \pm 14,8 \mathrm{aA}$ & $2,8 \pm 3,8 \mathrm{aA}$ & $13,8 \pm 11,3 \mathrm{aA}$ & $12,5 \pm 10,5 \mathrm{aA}$ & $37,8 \pm 12,3 \mathrm{aA}$ \\
\cline { 2 - 7 } & $\mathrm{SR}$ & $14,2 \pm 14,4 \mathrm{aA}$ & $2,2 \pm 3,4 \mathrm{aA}$ & $7,5 \pm 7,6 \mathrm{aA}$ & $6,8 \pm 5,9 \mathrm{aA}$ & $28,2 \pm 18,7 \mathrm{aA}$ \\
\hline
\end{tabular}

Médias seguidas por letras minúsculas distintas na linha diferem entre si $(\mathrm{P}<0,05)$.

Médias seguidas por letras maiúsculas distintas na coluna diferem entre si $(\mathrm{P}<0,05)$.

CR:congelação rápida com resfriamento lento; SR:congelação rápida sem resfriamento lento. D1:10\% de gema de ovo e acréscimo de $50 \%$ de açúcares e tampões em relação ao D5; D2:10\% de gema de ovo e redução de $50 \%$ de açúcares e tampões em relação ao D5; D3:20\% de gema de ovo e acréscimo de 50\% de açúcares e tampões em relação ao D5; D4:20\% de gema de ovo e redução de 50\% de açúcares e tampões em relação ao D5; D5:meio diluidor semelhante ao proposto por Martin et al. (1979), com 20\% de gema de ovo, lactose-glicose-EDTA.

\section{DISCUSSÃO}

A motilidade progressiva foi a única característica que variou significativamente entre os crioprotetores intracelulares utilizados. Os melhores resultados foram encontrados nos meios contendo acetamida do que naqueles com etilenoglicol, dependendo do diluidor utilizado. Essas semelhanças na capacidade de crioproteção entre crioprotetores intracelulares em meios de congelação de sêmen eqüino com a mesma composição de açúcares, tampões e gema de ovo e/ou leite também foram encontradas por Graham (2000), Medeiros et al. (2002) e Vidament et al. (2002). Parece que muitos agentes crioprotetores são eficazes durante a congelação de sêmen eqüino, podendo substituir o glicerol sem prejuízo aos espermatozóides durante a criopreservação.

Quanto às características espermáticas, não houve alteração naquelas avaliadas (motilidade, vigor espermático e integridade funcional (HO) da membrana plasmática dos espermatozóides) após a descongelação com a redução da concentração de gema de ovo de $20 \%$ para $10 \%$, nos diluidores com menor (D2 e D4) e maior (D1 e D3) concentração de açúcares e tampões, independentemente do crioprotetor intracelular utilizado. Quanto à quantidade de fosfolipídios, a proporção contida em $10 \%$ de gema de ovo $(1,1 \%)$ já está acima da considerada ótima (entre 0,5\% e 1,0\%) por Pickett e Berndtson (1978). A concentração de gema de ovo nos diluidores de congelação de sêmen eqüino tem variado de $2 \%$ (Tischner, 1979; Palmer, 1984; Ecot et al., 2000; Vidament et al., 2001) a 20\% (Martin et al., 1979; Braun et al., 1995), e sua substituição por componentes protéicos, como soro eqüino ou soro bovino ou albumina sérica bovina, nos diluidores usados para a congelação de sêmen eqüino, não tem proporcionado melhor efeito crioprotetor aos espermatozóides que a gema de ovo sozinha (Angola e Quintero, 1996) ou associada ao leite (Jasko et al., 1992; Braun et al., 1995; Juliani et al., 2002). Com tais 
resultados, a redução na porcentagem de espermatozóides estruturalmente íntegros observada em tratamentos contendo $10 \%$ de gema parece ser mais resultante da queda da osmolaridade ou composição do meio que o efeito da redução de gema de ovo, haja vista que meios com $10 \%$ de gema, mas com osmolaridade acima do diluidor-controle, mostraram-se igualmente eficientes em preservar a integridade estrutural das membranas espermáticas.

Independentemente da quantidade de gema de ovo, do crioprotetor intracelular utilizado, diluidores com maior concentração de açúcares e tampões (D1 e D3) e o diluidor-controle lactoseEDTA-gema de ovo (D5) preservaram melhor $(\mathrm{P}<0,05)$ a integridade estrutural das membranas plasmática e acrossomal dos espermatozóides do que os diluidores com uma menor concentração de açúcares e tampões (D2 e D4). Ficou evidente, assim, que, para os crioprotetores intracelulares e curvas de resfriamento utilizadas, a redução das quantidades de açúcares e tampões comprometeu a preservação da viabilidade espermática pós-descongelação. Essa influência foi significativa quando da análise da integridade estrutural da membrana e mostrou uma tendência consistente nos outros parâmetros avaliados. Considerando a preservação da integridade das membranas como um sinal que as demais estruturas presentes na cabeça também seriam preservadas na mesma intensidade, como, por exemplo, o núcleo, que é importante no processo de fertilização, os diluidores que apresentaram menor concentração de crioprotetores extracelulares (D2 e D4) demonstraram pouco potencial para serem utilizados na criopreservação de sêmen eqüino. A queda da viabilidade pode ser conseqüência da queda da osmolaridade do diluidor, resultante da redução nas quantidades dos crioprotetores extracelulares.

Faz-se necessário ressaltar que a composição adequada de um diluidor seminal não depende, apenas, de uma adequada concentração de crioprotetores intracelulares e extracelulares, mas da combinação ideal das substâncias que são utilizadas, levando-se em consideração qual a melhor fonte de energia para os espermatozóides, as melhores substâncias não iônicas, o tipo de macromoléculas e a concentração e o tipo ideal de crioprotetor intracelular. Isso porque, segundo Keith (1998), a presença de determinados açúcares, dependendo da concentração dessas substâncias nos diluidores de congelação de sêmen eqüino, influiu negativamente sobre a viabilidade espermática.

Além dos açúcares (glicose e lactose), os diluidores utilizados no presente experimento continham diferentes concentrações de substâncias tampões (citrato de sódio, bicarbonato de sódio e EDTA). Essas substâncias estavam em menor concentração nos piores meios (D2 e D4). De acordo com Bogart e Mayer (1950), existe uma aparente associação entre o grau de ionização dos sais que compõem os diluidores e os efeitos deletérios destes sais sobre os espermatozóides. Dessa maneira, parece que a sobrevivência espermática durante $\mathrm{O}$ armazenamento em baixas temperaturas, aparentemente, está inversamente relacionada com o grau de ionização desses sais. Aqueles pesquisadores, ao compararem algumas substâncias tampões, verificaram que o citrato de $\mathrm{Na}$ tem o menor índice de ionização, sendo considerado ótimo agente tampão. Segundo Silva et al. (1996), agentes crioprotetores extracelulares como as substâncias tampões podem agir sinergicamente com os crioprotetores intracelulares, propiciando ambiente adequado aos espermatozóides durante a congelação/descongelação. Além disso, a atividade metabólica dos espermatozóides causa aumento na concentração de íons hidrogênio no meio extracelular. Caso não exista nenhum mecanismo de remoção desses íons, ocorre redução do $\mathrm{pH}$ do meio onde os espermatozóides se encontram, acarretando diminuição na longevidade e fertilidade dessas células (Smith, 1984, citado por England, 1993).

O EDTA, que está presente no diluidor lactosegema, sendo um quelante de cálcio (Graham, 1996), reduz a concentração deste íon no ambiente extracelular, o que, provavelmente, pode contribuir para minimizar a entrada de cálcio nos espermatozóides. Durante a criopreservação, as membranas dos espermatozóides sofrem mudanças na fluidez, semelhantes às que ocorrem durante a capacitação natural, tornando-as permeáveis aos íons cálcio que promovem tanto a capacitação como a reação acrossômica. Esses dois eventos limitam a sobrevivência dos espermatozóides por tempo prolongado, porque, nesse estágio, os espermatozóides estão preparados para fertilizar 
o ovócito e, quando a fertilização não acontece em um curto período de tempo, os espermatozóides não resistem e morrem (Watson, 1995). Pode também ter sido causa da queda na viabilidade espermática pósdescongelação, encontrada nos diluidores D2 e $\mathrm{D} 4$, a inadequada proporção de citrato de $\mathrm{Na}$, EDTA e bicarbonato de $\mathrm{Na}$ presente nesses meios.

O diluidor com alta concentração de açúcares, tampões e sais e $20 \%$ de gema mostrou potencial para proteção da característica integridade estrutural das membranas plasmática e acrossomal. Este achado é encorajador e abre uma janela para estudos complementares de avaliação do efeito da osmolaridade dos diluidores sobre a preservação da viabilidade espermática pós-descongelação.

\section{REFERÊNCIAS BIBLIOGRÁFICAS}

ANGOLA, A.P.; QUINTERO, L.Z. Efecto de la sustitución de yema de huevo por albúmina sérica bovina, suero eqüino o suero bovino en el diluyente de congelación sobre la viabilidad posdescongelación del espermatozoide eqüino. Vet. Mexico, v.27, p.221-227, 1996.

BOGART, R.; MAYER, D.T. The effects of egg yolk on the various physical and chemical factors detrimental to spermatozoan viability. J. Anim. Sci., v.9, p.143-152, 1950.

BRAUN, J.; HOCHI, S.; OGURI, N. et al. Effect of different protein supplements on motility and plasma membrane integrity of frozen-thawed stallion spermatozoa. Cryobiology, v.32, p.487492, 1995.

BURNS, P.J. Modification of kenney's extender for cryopreservation of equine spermatozoa. In: INTERNATIONAL CONGRESS ON ANIMAL REPRODUCTION, 12., 1992, Den Haagen. Proceedings... Den Haagen, 1992. p.1849-1851.

BURNS, P.J.; REASNER, D.S. Computerized analysis of sperm motion: effects of glycerol concentration onthe cryopreservation of equine spermatozoa. J. Equine Vet. Sci., v.15, p.377380, 1995.

CROWE, J.H.; CROWE, L.M.; CARPENTER, J.F. et al. Stabilization of dry phospholipid bilayers and proteins by sugars. Biochem. J., v.242, p.1-10, 1987.

ECOT, P.; VIDAMENT, M.; MONARC, A. et al. Freezing of stallion semen: interactions among cooling treatments, semen extenders and stallions. J. Reprod. Fertil., v.56, suppl., p.141$150,2000$.

ENGLAND, G.C.W. Cryopreservation of dog semen : a review. J. Reprod. Fertil., v.47, suppl., p.243-255, 1993.

GOMES, G.M.; PAPA, F. O.; JACOB, J.C.F. et al. Melhoria dos parâmetros espermáticos pósdescongelação com o meio MP 50 para sêmen de garanhões da raça mangalarga machador. Rev. Bras. Reprod. Anim., v.26, p.187-189, 2002.

GRAHAM, J.K. Cryopreservation of stallion spermatozoa. Vet. Clin. N. Am.: Equine Pract., v.12, p.131-145, 1996.

GRAHAM, J.K. Evaluation of alternative cryoprotectants for preserving stallion spermatozoa. In: INTERNATIONAL CONGRESS ON ANIMAL REPRODUCTION, 14., 2000. Proceedings... Stockholm, 2000. v.2, p.307.

HARRISON, R.A.P.; VICKERS, S.E. Use of fluorescent probes to assess membrane integrity in mammalian spermatozoa. J. Reprod. Fertil., v.88, p.343-352, 1990.

JASKO, D.J.; HATHAWAY, J.A.; SCHALTENBRAND, W.D. et al. Effect of seminal plasma an egg yolk on motion characteristics of cooled stallion spermatozoa. Theriogenology, v.37, p.1241-1252, 1992.

JULIANI, G.C.; HENRY, M.; MIRON, D.M. et al. Congelamento de sêmen eqüino: efeito de diferentes crioprotetores e diluidores. In: CONGRESSO BRASILEIRO DE MEDICINA VETERINÁRIA, 29., 2002, Gramado, RS Anais...Gramado: Combravet, 2002.

KEITH, S.L. Evaluation of new cryoprotectans for the preservation of equine spermatozoa. 1998. 104f. Thesis (Master of Science) Colorado State University, Colorado.

MARTIN, J.C.; KLUG, E.; GÜNZEL, A.R. Centrifugation of stallion semen and its storage in large volumes straws. J. Reprod. Fertil., v.27, suppl., p.47-51, 1979. 
MANUAL para Exame Andrológico e Avaliação de Sêmen Animal. 2.ed. Colégio Brasileiro de Reprodução Animal, Belo Horizonte, 1998. p.2527.

MEDEIROS, A.S.L.; GOMES, G.M.; CARMO, M.T. et al. Cryopreservation of stallion sperm using different amides. Theriogenology, v.58, p.273-276, 2002.

MELO, M.I.V; HENRY, M. Teste hiposmótico na avaliação do sêmen eqüino. Arq. Bras. Med. Vet. Zootec., v.51, p.71-78, 1999.

NEVES NETO, J.R.; PAPA, F.O.; LEME, D.P. Avaliação entre congelabilidade e fertilidade do sêmen eqüino frente a dois diluidores. Rev. Bras. Reprod. Anim., v.23, p.301-302, 1999.

PALMER, E. Factors affecting stallion semen survival and fertility. In: INTERNATIONAL CONGRESS ANIMAL REPRODUCTION AND ARTIFICIAL INSEMINATION, 10., 1984. Proceedings... Urbana, 1984. p.377.

PAPA, F.O.; TAVARES, C.V.N.; MEIRA, C. et al. Glicina-gema: proposta de um novo diluidor para a congelação de sêmen bovino. In: CONGRESSO BRASILEIRO DE REPRODUÇÃO ANIMAL, 10., 1993, Belo Horizonte. Anais... Belo Horizonte: CBRA, 1993. p.313.

PAPA, F.O.; ZAHN, F.S.; DELL'ÁQUA JÚNIOR, J.A. et al. Utilização do diluente MP 50 para a criopreservação de sêmen eqüino. Rev. Bras. Reprod. Anim., v.26, p.184-187, 2002.

PICKETT, B.W.; BERNDTSON, W.E. Principles and techniques of freezing spermatozoa. In: SALISBURY, G.E., VANDEMARK, J.R. (Eds.). Physiology and reproduction and artificial insemination of cattle. San Francisco: Freeman, 1978. p.494.
SAMPAIO, I.B.M. Estatística aplicada a experimentação animal. Belo Horizonte: Fundação de Ensino e Pesquisa em Medicina Veterinária e Zootecnia, 1998. 221p.

SAMPER, J.C.; HELLANDER, J.C.; CRABO, B.G.. Relationship between the fertility of fresh and frozen semen and semen quality. J. Reprod. Fertil., v.44, suppl.,p.107-114, 1991.

SILVA, AL.A.; YAMASAKI, R; DE SALA, M.M et al. The addition of fructose or sodium citrate does not improve recovery rates of cryopreserved human spermatozoa. Int. J. Fertil., v.41, p.304-309, 1996.

TISCHNER, M. Evaluation of deep-frozen semen in stallions. J. Reprod. Fertil., v.27, suppl., p.53-59, 1979.

SISTEMA de análise estatística e genética. Versão 6,0. SAEG. Viçosa, MG:UFV, 1997.

VIDAMENT, M.; DAIRE, C.; YVON, J.M. et al. Motility and fertility of stallion semen frozen with glycerol and/or dimethyl formamide. Theriogenology, v.58, p.249-251, 2002.

VIDAMENT, M.; YVON, J.M.; COUTY, I. Et al. Advances in cryopreservation in modified INRA 82. Anim. Reprod. Sci., v.68, p.201-218, 2001.

WATSON, P.F. Recent developments and concepts in cryopreservation of spermatozoa and the assessment of their post-thawing function. Reprod. Fertil. Dev., v.7, p.871-891, 1995.

ZÚCCARI, C.E.S.N. Efeito da criopreservação sobre a integridade estrutural da célula espermática eqüina. 1998. 121f. Tese (Doutorado em Medicina Veterinária) Faculdade de Medicina Veterinária e Zootecnia, Universidade Estadual Paulista, Botucatu, SP. 